\title{
Multiplexing optical fiber Fabry-Perot interferometers based on air-microcavities
}

\author{
R.A. Perez-Herrera ${ }^{* a}$, S. Novais ${ }^{b}$, M. Bravo ${ }^{a}$, D. Leandro ${ }^{a}$, S.F. Silva ${ }^{b}$, O. Frazão ${ }^{b}$, M. Lopez-Amo ${ }^{a}$ \\ ${ }^{a}$ Dept. of Electrical Electronic and Communication Engineering and Institute of Smart Cities (ISC), \\ Public University of Navarra, 31006 Pamplona, Spain; \\ ${ }^{\mathrm{b}}$ INESC TEC-Institute for Systems and Computer Engineering, Technology and Science, Rua do \\ Campo Alegre 687, 4150-179, Porto, Portugal
}

\begin{abstract}
In this work we demonstrate the multiplexing capability of new optical fiber Fabry-Perot interferometers based on airmicrocavities using a commercial FBG interrogator. Three optimized air-microcavity interferometer sensors have been multiplexed in a single network and have been monitored using the commercial FBGs interrogator in combination with FFT calculations. Results show a sensitivity of $2.18 \pi \mathrm{rad} / \mathrm{m} \varepsilon$ and a crosstalk-free operation.
\end{abstract}

Keywords: Fabry-Perot interferometers, optical fiber sensor multiplexing, FFT, air-microcavity.

\section{INTRODUCTION}

Nowadays, the measurement of axial strain using optical fiber sensors is a common application. Point and distributed strain sensors are used for civil works monitoring, aerospace applications and mechanical vibrations measurements, among others [1,2]. Recently, in-fiber air-microcavities have shown a strain sensitivity higher than an order of magnitude compared with well stablished point sensors such as fiber Bragg gratings (FBGs) or long period gratings (LPGs) [3]. In many applications, several strain measurements have to be placed along a structure to correctly measure its deformation. Usually, distributed sensing or alternatively FBGs sensors are used for this task. FBGs are very well-suited optical fiber sensors because the measured parameter is encoded in the wavelength shift, immune to amplitude variations of the optical source or induced attenuations in the fiber. Because of this, optical interrogators based on a tunable laser and a synchronized optical filter are the most successful monitoring technology for FBGs sensors [4]. These wide-spread interrogators are ideally designed to multiplex FBG sensors using wavelength-division multiplexing. However, signal processing can be applied to adapt the interrogator for real-time monitoring of interferometric sensors [5]. In this work we experimentally demonstrate the multiplexing capabilities of in-fiber air-microcavities for strain measurements, showing a sensitivity of $2.18 \pi \mathrm{rad} / \mathrm{m} \varepsilon$ without crosstalk. These measurements have been carried out using a commercial interrogator initially designed for FGB sensors measurements, by means of a home designed software suitable for FFT calculations of the acquired optical spectrum at interrogation speeds up to $1 \mathrm{~Hz}$.

\section{EXPERIMENTAL SETUP}

\subsection{Fabrication process}

As aforementioned, three air-microcavity based interferometric sensors have been manufactured and multiplexed in this study. The sensing cavities were produced by fusion splicing a small section of silica tube (hollow-core fiber) with 57 and $125.5 \mu \mathrm{m}$ of internal and external diameters respectively, between two sections of standard single mode fiber (SMF 28e). In Figure 1, a scheme of the sensors used and a microscope photograph of the sensor are presented. The fiber and the silica tube were placed in the splicing machine in manual operation with a lateral offset, to ensure that the arc discharge was mainly applied in the SMF region, preventing the collapsing of the silica tube region. The silica tube was then cleaved with the desired length, under a $5^{\times}$magnifying lens. The microcavity volume, accordingly the free-spectral range (FSR) of the interferometer, can be tailored by applying successive electrical arcs [6]. In order to avoid crosstalk 
and allow a correct identification of the sensors in the FFT domain, three sensors have been manufactured with different cavity sizes: $\sim 60,105$ and $165 \mu \mathrm{m}$ for sensor 1 to sensor 3 (S1 to S3). Consequently, the measured FSRs of the sensors are $20,11.5$ and $7.3 \mathrm{~nm}$ respectively for $\mathrm{S} 1$ to $\mathrm{S} 3$. The independent spectral characterization of the sensors can be seen in Figure 2.

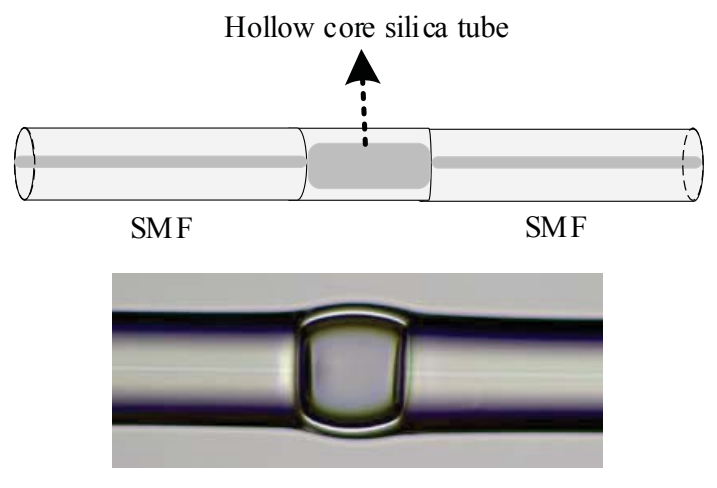

Figure 1. Schematic and microscope image of a sensor head.

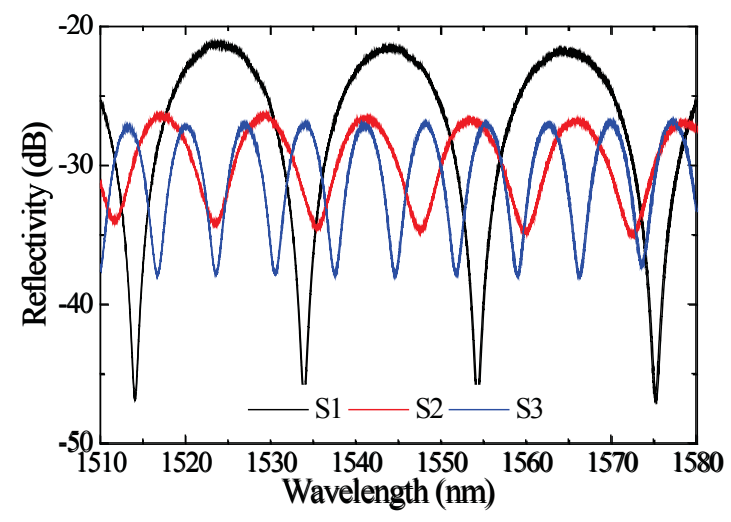

Figure 2. Reflected spectra of the three micro-air FabryPerot sensors independently.

\subsection{Experimental setup and working principle}

Figure 3 shows a schematic diagram of the experimental setup for the strain sensors multiplexing. The setup is formed by a commercial FBG sensors interrogator (Micron Optics ${ }^{\circledR}$ SM 125), a $2 \times 4$ fiber-optic coupler (OC) and three airmicrocavities strain sensors connected to three output ports of the coupler. One of the sensors was placed on a motorized translation stage with a maximum resolution of $17 \mathrm{~nm}$. The characterization of the sensor has been performed by applying an axial strain of $500 \mu \varepsilon$ in steps of $3.5 \mu \varepsilon$ each.

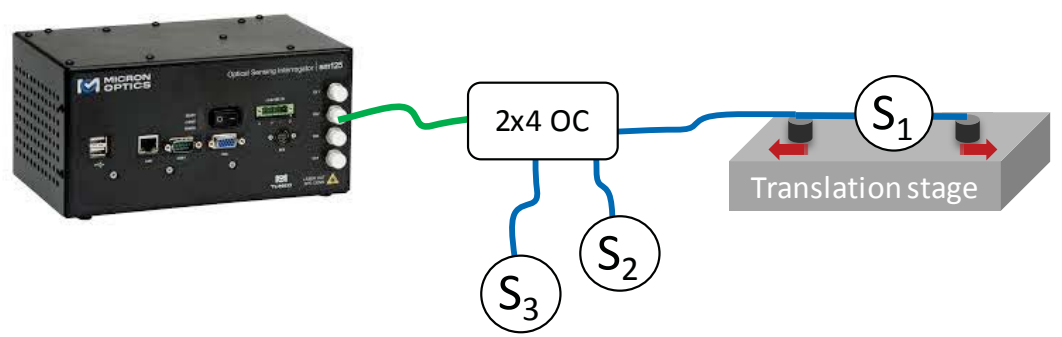

Figure 3. Schematic diagram of the experimental setup.

The interrogation of the sensors has been carried out using a single channel of the optical interrogator, which is connected to one port of the optical coupler. In this manner, the FBG interrogator records the combined interferometric signal reflected by the air-microcavity Fabry-Perot sensors at a refresh rate of $1 \mathrm{~Hz}$ (Figure 3). In order to perform the measurement of the sensors in the FFT domain in real-time, a custom-made control software was employed. The software logs the reflected spectra of the sensors, performs the FFT, and moves the translation stage.

The principle of operation of this multiplexing technique is based on the combination of the received spectra at the optical coupler and its FFT analysis. The combined spectrum is depicted in Figure 4. To separate each sensor information, the FFT of the spectrum is analyzed obtaining the contributions presented in Figure 5. There, it can be clearly identified the independent contributions of the three sensors at the spatial frequencies of $0.05,0.0875$ and 1.375 $\mathrm{nm}^{-1}$ for $\mathrm{S} 1$ to $\mathrm{S} 3$ respectively. In order to monitor the sensor changes, the phase of the FFT is evaluated at the spatial 
frequencies that correspond to each sensor. In this manner, these phase variations reflect the wavelength shift induced by the strain in each spectral contribution independently.

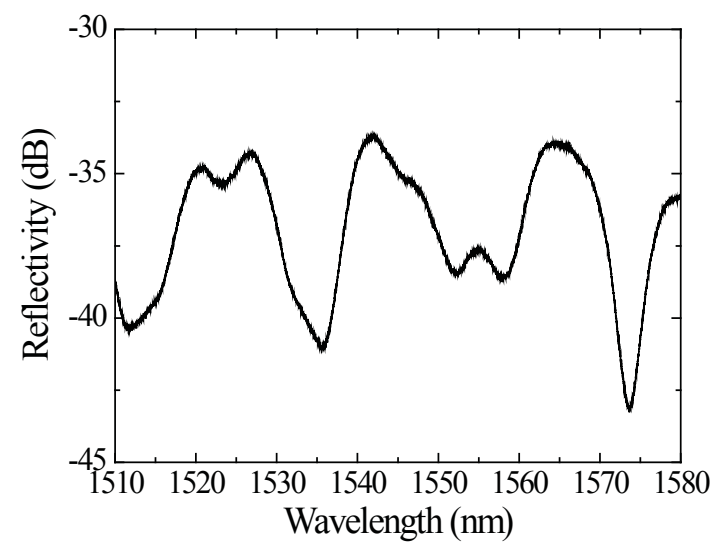

Figure 4. Combined spectrum of the multiplexed sensors.

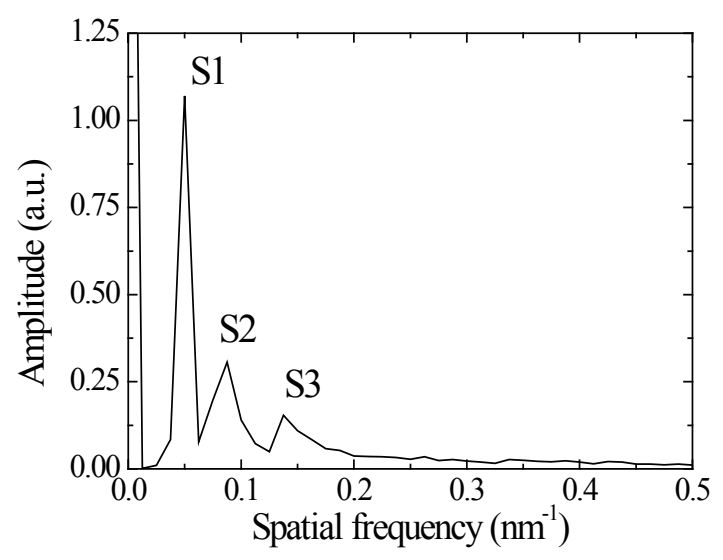

Figure 5. Magnitude of the FFT from the spectrum of Figure 4.

\section{RESULTS AND DISCUSSION}

Figure 6 shows the FFT-phase variations of the sensors when the strain characterization is performed in S1. The sensor presents a clear linear response with sensitivity of $2.18 \pi \mathrm{rad} / \mathrm{m} \varepsilon$ and an error $\mathrm{R}^{2}=0.99992$. It can also be seen in the figure that S2 and S3 remained unaltered during the test, demonstrating the crosstalk-free operation of the proposed multiplexing system.

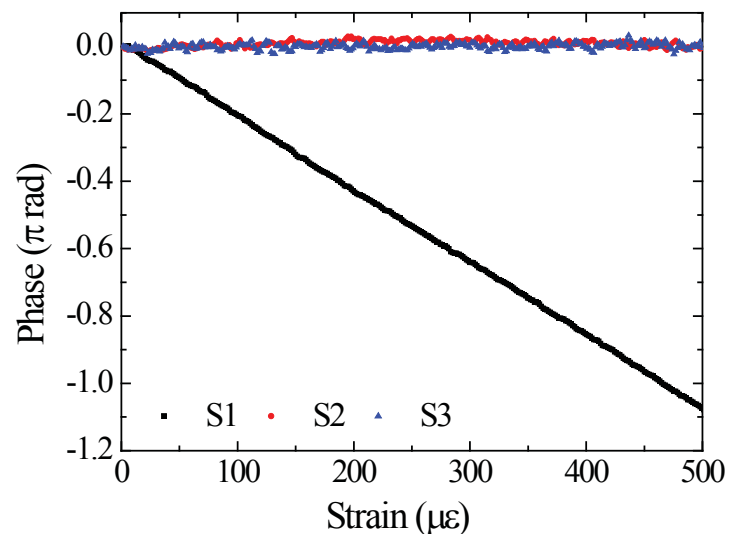

Figure 6. Characterization of S1 while S2 and S3 are not strained.

Finally, figure 7 presents the stability of the sensors with time for a 10 minutes-test under static conditions. The stability values obtained present a system resolution of $4.5 \mu \varepsilon$ for S1 and $15 \mu \varepsilon$ for S2 and S3. It is interesting noticing that the values of the sensors vary accordingly with the FFT-amplitude of each sensor system. Although it is a topic that should be studied in depth, it was observed that it exists a direct correlation between the phase noise and the amplitude of its corresponding contribution amplitude (proportional to the finesse of the resonator). Hence, it can be inferred that higher finesse values allow better resolution of the system. 

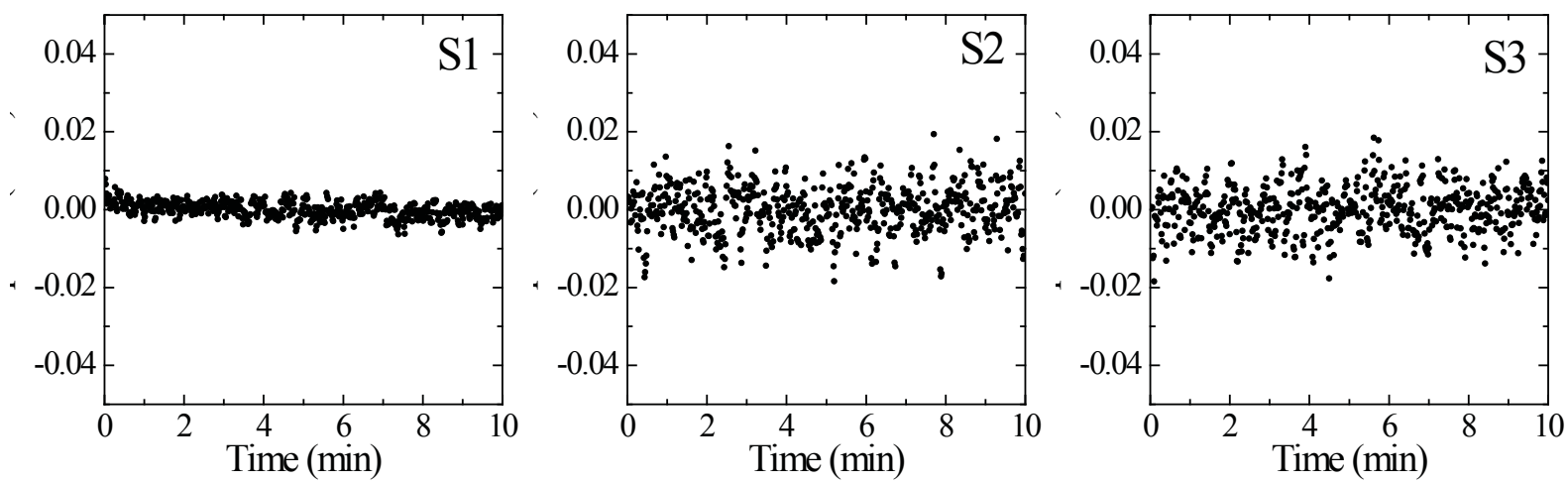

Figure 7. 10-minutes stability measurements for sensors $\mathrm{S} 1$ to S3.

\section{CONCLUSIONS}

In this work, three air-microcavity Fabry-Perot strain sensors were successfully multiplexed by using the FFT method. A sensitivity of $2.18 \pi \mathrm{rad} / \mathrm{m} \varepsilon$ was measured showing crosstalk-free operation. Compared with [5], the obtained system resolution is approximately $35 \%$ higher. Besides, the multiplexing capability can be extended by tailoring the FSR of the resonators and placing them at independent zones of the spatial-frequency spectrum. Also, bus multiplexing structures can be further studied. In that case, the structure would be coupler-free and, theoretically, would provide the same performance. Finally, a homogenization of the fabrication process will be developed with the aim of obtaining similar resonator finesses and, thus, similar spectral contributions and sensor resolution.

\section{ACKNOWLEDGMENT}

This work was supported by the Spanish AEI TEC2016-76021-C2, FEDER Funds; and also by the ERDF - European Regional Development Fund through the Operational Programme for Competitiveness and Internationalization COMPETE 2020 Programme and by National Funds through the Portuguese funding agency, FCT - Fundação para a Ciência e a Tecnologia within project ENDOR - Endoscope based on New Optical Fibre Technology for Raman Spectroscopy (POCI-01-0145-FEDER-029724). M. Bravo received funding from Marie Skłodowska-Curie grant agreement No 838143.

\section{REFERENCES}

[1] Alexis, M. [Fiber Optic Sensors: Fundamentals and Applications.] Press Monographs, (2015).

[2] Islam, M., R. et al., "Chronology of Fabry-Perot Interferometer Fiber-Optic Sensors and their applications: A Review," Sensors 14, 7451-7488 (2014).

[3] Shen L. et al., "High-sensitivity strain sensor based on in-fiber rectangular air bubble," Scientific Reports 5, 7624 (2015).

[4] Zhang, Y. et al., "Application of FBG sensing technique for monitoring and early warning system of highspeed railway track conditions", 25th Optical Fiber Sensors Conference (OFS), 1-4 (2017).

[5] Daniel, L. et al., "Real-Time FFT Analysis for Interferometric Sensors Multiplexing," J. Lightwave Technol. 33, 354-360 (2015).

[6] S. Novais, et al., "Lateral Load Sensing With an Optical Fiber Inline Microcavity," IEEE Photonics Technology Letters, 29(17), 1502-1505, (2017). 